
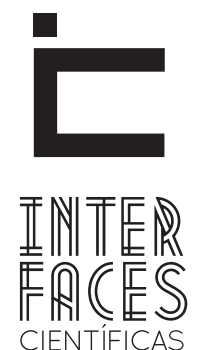

EDUCAÇÃO

ISSN IMPRESSO 2316-333X

ISSN ELETRÔNICO 2316-3828

PARTE 1 - FUNDAMENTOS E DEBATES ATUAIS DE PEDAGOGIA SOCIAL

\title{
FUNDAMENTOS DE LA PEDAGOGíA SOCIAL Y DE LA EDUCACIÓN SOCIAL
}

Gloria Pérez Serrano ${ }^{1}$

Ana Fernández-García ${ }^{3}$
José Luis García Llamas

\section{RESUMEN}

Este artículo estudia los fundamentos de la Pedagogía Social (PS) y de la Educación Social (ES). Se analizan las diferencias y semejanzas entre conceptos afines. Resulta arduo fundamentar la Pedagogía Social como ciencia y la Educación Social como ámbito de conocimiento, dado que toda realidad es compleja, y no puede ser aprehendida con herramientas simples. Los conceptos que se manejan son polivalentes y con fronteras no suficientemente delimitadas, dado que interactúan y se interrelacionan con otras disciplinas que trabajan en el campo de 10 social. La Pedagogía Social se construye y reconstruye continuamente de modo abierto, es versátil, cambiante y viva. Es un concepto complejo. De ahí que se insista en la necesidad de la investigación teórica y en la reflexión sobre la práctica. La Educación Social presenta dos perspectivas: una que va desde la vertiente normalizada que es el proceso de socialización de los individuos, y el continuum que va desde la infancia a la edad adulta. Y otra, desde la óptica de la educación especializada, que hace referencia al tratamiento e intervención de todo tipo de inadaptados sociales. Se estudian conceptos vinculados con la Pedagogía Social: Educación Social, Trabajo Social y Sociología de la Educación. A modo de síntesis se muestran los elementos de -concepto, objeto, método y ámbito- que configuran cada uno de los constructos señalados. Estos conceptos se interrelacionan y complementan, por lo que las fronteras existentes entre ellos se desdibujan, lo que dificulta su delimitación conceptual.

\section{PALABRAS-CLAVE}

Pedagogía Social. Educación Social. Fundamentos científicos. Trabajo Social. Sociología de la Educación 


\section{ABSTRACT}

This article studies the fundamentals of social pedagogy (PS) and social education (ES). The diferences and similarities are analized among related concepts. It is hard to base the Social Pedagogy as a science, and social education as a field of knowledge, because every reality is complex, and it cannot be apprehended with simple tools. The concepts that are addressed are multipurpose and poorly defined borders, as they interact and interrelate with other disciplines working in the "social" field. Social Pedagogy is continuously constructed and reconstructed open way, it's versatile, changing and alive. It is a complex concept. Hence it insists on the $y$ theoretical investigation in reflection on practice. The Social Education presents two perspectives. One, from the normalized slope: the socialization of individuals, this continuum from chil- dhood to adulthood. And another, from the perspective of special education, which refers to treatment and involvement of all sorts of social marginalized. Related concepts are studied between them and with the Social Pedagogy: Social Education, Social Work and Sociology of Education. To summarize the elements of concept, object, method and field that shaping each of the indicated constructs are shown. These concepts are interrelated and complementary, so that the boundaries between them are blurred, making it difficult conceptual delimitation.

\section{KEYWORDS}

Social Pedagogy. Social Education. Scientific Foundations. Social Work. Sociology of Education.

\section{RESUMO}

Este artigo discute os fundamentos da Pedagogia Social (PS) e da Educação Social (ES). São analisadas as diferenças e semelhanças entre conceitos afins. Resulta difícil fundamentar a Pedagogia Social como ciência e a Educação Social como âmbito de conhecimento, dado que toda realidade é complexa e não pode ser apreendida com ferramentas simples. Os conceitos que se abordam são polivalentes e com fronteiras insuficientemente delimitadas, uma vez que interagem e interrelacionam com outras disciplinas que trabalham no campo "do social". A Pedagogia Social se constrói e reconstrói continuamente de modo aberto, é versátil, cambiante e viva. É um conceito complexo. Daí que se insiste na necessidade da investigação teórica y na reflexão sobre a prática. A Educação Social apresenta duas perspectivas. Uma, a partir a vertente normalizada: o proceso de socializaão dos individu- os, esse continuum que vai desde a infância até a idade adulta. E outra, a partir da ótica da educação especializada, que faz referência ao tratamento e intervenção de todo tipo de marginalizados sociais. São estudados os conceitos vinculados entre si e com a Pedagogia Social: Educação Social, Trabatho Social e Sociologia da Educação. A modo de síntese, são mostrados os elementos de conceito, objeto, método e âmbito que configuram cada um dos construtos assinalados. Estes conceitos se interrelacionam e se complementam, de modo que as fronteiras existentes entre eles se dissolvem, o que dificulta a sua delimitação conceitual.

\section{PALAVRAS-CHAVE}

Pedagogia Social. Educação Social. Fundamentos científicos. Trabalho Social. Sociologia da Educação. 


\section{INTRODUCCIÓN A LA PEDAGOGÍA SOCIAL Y EDUCACIÓN SOCIAL}

Este artículo estudia los fundamentos de la Pedagogía Social (PS) y de la Educación Social (ES). Se analizan también las diferencias y semejanzas entre conceptos afines.

En la actualidad, las ciencias que se dedican al estudio de lo social parece que gozan de buena salud. Esta revitalización se debe a un conjunto de causas que ponen en valor lo social. Fundamentar, hoy, la Pedagogía Social como ciencia y la Educación Social como ámbito de conocimiento, resulta arduo, dado que toda realidad es compleja y no puede ser aprehendida con herramientas simples.

La Pedagogía Social se construye continuamente de modo abierto, es versátil y dinámica. De ahí que se insista en la necesidad de la investigación teórica, así como, en la reflexión sobre la práctica.

\subsection{FUNDAMENTOS DE PEDAGOGÍA SOCIAL [PS]}

Pedagogía Social es una disciplina científica, teórico-aplicada. Se sitúa en el grupo de las Ciencias de la Educación, y la praxis ejercida en sus múltiples formas, se realiza por los denominados pedagogos sociales.

Este constructo está compuesto por dos términos: "pedagogía" y "social". Pedagogía hace referencia tanto al conocimiento como a la acción, y también a la técnica o tecnología de la Educación Social. Social, hace referencia al "sujeto en relación", en grupos y comunidades, en situaciones normales o conflictivas, de necesidad o de desvalimiento.

Se va configurando en una doble dimensión. Por una parte aborda la dimensión integradora de lo epistemológico, lo tecnológico y lo normalizado y, por otra, las situaciones de inadaptación, exclusión y conflicto social que da lugar a una Pedagogía Social especializada.

\subsection{CONCEPTO DE PEDAGOGÍA SOCIAL}

En el devenir histórico de la Pedagogía Social se han desarrollado concepciones referidas tanto a su dimensión teórica como aplicada.

El estudio de escuelas y autores, contextos geográficos y problemas, principios de actuación y pautas de investigación, es una fuente de inspiración para comunidad científica preocupada por la Pedagogía Social (PS). Se va configurando no sólo desde la praxis pedagógica, sino también desde instancias teóricas y metateóricas que, desde otros contextos van ayudando a conformar un cuerpo de conocimiento básico para afianzar esta ciencia. Algunos reclaman que se reserve la expresión Pedagogía Social para referirse a los movimientos alemanes en torno a la década de 1920, que, en razón de su coyuntura histórica, vinculaba la ayuda a los desvalidos, necesitados y abandonados.

\subsubsection{NUESTRO CONCEPTO DE PEDAGOGÍA SOCIAL.}

Llegados a este punto nos atrevemos a ofrecer nuestra definición: es una ciencia teórico-aplicada a la mejora de individuos y grupos, con carácter propositivo. Se ocupa de la prevención, la ayuda y reinserción de todos los que padecen algún tipo de deficiencia o de disocialización, orientada a la mejora de la calidad de vida desde una perspectiva aplicada, tanto en la vertiente normalizada como en la especializada. Tiene los siguientes rasgos definitórios:

\section{Ciencia teórico-aplicada}

Al considerar la Pedagogía Social como ciencia, nos indica que es tarea suya obtener conocimientos científicos en su campo de saber, con el fin de actuar en la realidad y verificar dicha fundamentación en la praxis, como conocimiento y acción, investigación e intervención, ciencia y tecnología. 


\section{Carácter propositivo}

Este carácter exige asumir una perspectiva analítica y evaluativa respecto de una situación determinada, por lo que se realizan acciones de forma reflexiva y crítica, no precipitada. Es una ciencia pedagógico-social, práctica y aplicada, que se orienta a la intervención y a la mejora de individuos y grupos, con un carácter prescriptivo. Es intencional y trata de comprender la acción social.

Se ocupa de la prevención, la ayuda y re-inserción de todos los que padecen algún tipo de deficiencia o de disocialización

El destinatario de la PS es el ser humano a lo largo del ciclo vital, tanto en su dimensión individual como social. El objetivo es ayudar a individuos y grupos en la solución de coyunturas obstaculizadoras. Por una parte, orienta el desarrollo y aprendizaje social de los sujetos (socialización) y, por otra, previene las necesidades de relación, adaptación e inclusión (prevención y reintegración). Es una ciencia que se ocupa de la socialización del individuo y grupos, orientada a la mejora y transformación de la sociedad.

Orientada a la mejora de la calidad de vida

Al ser una ciencia social, considera lo social desde la vertiente de la educación, no sólo en su carácter descriptivo y asistencial, sino también con la perspectiva de mejora y perfeccionamiento.

Perspectiva aplicada tanto en la vertiente normalizada como especializada

La PS es una ciencia educativa-social que se desarrolla en espacios no formales, sin descuidar los procesos de socialización normalizados. Al ser una ciencia del espíritu, uno de sus rasgos característicos es la comprensión de la realidad a fin de obtener un mejor conocimiento de la misma para intervenir cualificadamente. La perspectiva aplicada debe estar presente tanto en la vertiente de la PS normalizada, como en la especializada. La PS como ciencia se ambienta y radica en la vitalidad de las condiciones educativas de la cultura y vida social.

\subsection{CARÁCTER CIENTÍFICO DE LA PEDAGOGÍA SOCIAL}

En este punto nos vamos a referir al carácter científico de la PS. La evolución científica y humana precisa de sujetos con una buena formación teórica y un conocimiento del manejo de los métodos y técnicas de investigación. De ahí, que a un investigador se le pida, que desarrolle actitudes, tales como: curiosidad y admiración, exigencia, orden, sistematización y rigor en la búsqueda.

Se requiere de cuatro requisitos esenciales: formación teórica, conocer los métodos y técnicas, actitudes científicas y tener en cuenta la finalidad de la ciencia.

\subsection{OBJETO DE ESTUDIO DE LA PEDAGOGÍA SOCIAL}

El objeto de estudio de la PS es la «educación social». Por su parte, la PS se ocupa de la prevención, ayuda y reinserción de los sujetos en relación con su contexto, ámbitos propios de su intervención.

Para algunos autores el objeto de estudio de la PS es: Natorp (el hombre en comunidad); Luzuriaga (la relación entre educación y sociedad); Volpi (la sociedad educadora); Arroyo (la educación al servicio de la comunidad); Quintana; Ortega (la educación social normalizada y especializada).

En suma, formulado de uno u otro modo por los autores mencionados, el objeto de estudio de la PS hace referencia a la educación social y al estudio de las necesidades sociales y educativas, tanto desde la perspectiva normalizada como especializada.

Para que la PS sea considerada ciencia tiene que tener en cuenta estas tres perspectivas: la científica, la disciplinar y la profesional.

\subsubsection{PERSPECTIVA CIENTÍFICA}

La PS, como cualquier ciencia, es un saber en construcción y re-construcción constante. Para hacer valer su cientificidad tiene que prestar atención 
a una serie de rasgos de naturaleza epistemológica, metodológica, sistemática, rigurosa, contrastable, verificable, con terminología específica, etc.

La PS no es menos ciencia que lo pueden ser las Ciencias Naturales, sólo que constituye un cuerpo de conocimiento organizado de distinto modo, porque son diferentes sus puntos de partida y sus conclusiones. Las ciencias Exactas parten de supuestos, en tanto que las Ciencias Sociales parten de hechos y acontecimientos, dado que tienen al ser humano como su objeto y también sujeto de estudio.

\subsubsection{PERSPECTIVA DISCIPLINAR}

En general, el volumen de conocimiento se ha ido incrementando progresivamente debido al desarrollo científico-tecnológico. Este aumento es cada vez más rápido y constituye una gran esperanza para fomentar la calidad de vida de todos los seres humanos.

Estos avances científicos hacen difícil delimitar las fronteras de cada ciencia, más aún, cuando ese conocimiento surge interconectado entre diversas ciencias. Los conocimientos cada vez son más complejos por sus inter-acciones e inter-relaciones en el amplio campo del saber, por tanto, resulta problemático llegar a poner fronteras en cada una de las disciplinas específicas del mundo científico. La delimitación de los conocimientos se hace cada vez más ardua debido al solapamiento existente entre ellos, así como el número de disciplinas que se ocupan del mismo objeto de estudio. El principal desafío se encuentra en definir las condiciones de racionalidad de la ciencia, es este caso de la PS.

Hace falta investigación rigurosa y abundante, de carácter filosófico e histórico para evitar el solapamiento al que se hace referencia.

\subsubsection{PERSPECTIVA PROFESIONAL}

Los diferentes campos de la acción social reclaman profesionales con diferentes competen- cias para realizar tareas concretas y especializadas en su campo.

El pedagogo social requiere una formación teórico-científica-aplicada orientada a la construcción del conocimiento, la investigación y la intervención social. Es un experto cuya formación le capacita para fundamentar, justificar y comprender la acción educativa en la realidad social. Toda acción educativo-social ha de saber aplicar estos principios en cada una de las acciones que realice, sean de carácter preventivo, curativo y optimizador. Entre las funciones a realizar señalamos:

Analizar aspectos que conforman situaciones en diferentes contextos socio-educativos con el fin de intervenir y mejorar la realidad.

Anticipar con carácter preventivo, las situaciones de riesgo, vulnerabilidad y exclusión social.

Diagnosticar y planificar, a través de programas, acciones y proyectos para favorecer los procesos de socialización.

Realizar el seguimiento y evaluación de los programas, acciones y proyectos diseñados e implementados para cada contexto social.

Favorecer la resocialización y reinserción, terapia y curación, así como la protección y vigilancia de las conductas desviadas.

Con el desempeño de estas funciones, el pedagogo obtiene un conocimiento nomotético e ideográfico y utiliza la comprensión como método para interpretar la acción y las relaciones sociales. Precisa de la intervención del sujeto en ese proceso de comprensión.

El educador social precisa de una formación científico cultural que le otorgará una competencia personal y preparación tecnológica, orientada hacia el dominio de metodologías, técnicas y estrategias de 
intervención social. Debe estar especialmente preparado en el diseño de programas y proyectos socio-educativos. Por ello, "[] desde la educación social se debe formar a los futuros profesionales en competencias que les hagan capaces de proporcionar respuestas a las diferentes situaciones relacionadas con la igualdad/desigualdad, la inclusión/exclusión y la violencia" (BAS-PEÑA, PÉREZ-DE-GUZMÁN-PUYA, VARGAS, 2014, p. 10).

Al educador social, el Libro Blanco (ANECA, 2004, p.127) lo define como "Un agente de cambio social, dinamizador de grupos sociales a través de estrategias educativas que ayudan a los sujetos a comprender su entorno social, político, económico y cultural y a integrarse adecuadamente".

Estamos en condiciones de afirmar que la PS es una ciencia que tiene un corpus de conocimiento, hace uso del método científico en sus investigaciones dado que cuenta con un corpus de conocimiento, se ha organizado en una disciplina y prepara profesionales capacitados para actuar en diferentes ámbitos de la acción socioeducativa.

\section{FUNDAMENTOS DE LA EDUCACIÓN SOCIAL [ES]}

La Educación Social es una realidad que siempre ha estado implícita en la vida de los individuos y los pueblos. Se ha desarrollado en ágoras y plazas, en los espacios públicos, en diferentes asociaciones y organizaciones. En todos aquellos ámbitos comunitarios en los que fluye la vida en comunidad, por la interrelación de los unos con los otros. Este es el ámbito natural de la Educación Social, donde debe encontrar su renovado sentido. Si queremos comprender la Educación Social hemos de entender las fuerzas que modelaron su reciente historia.

\subsection{CONCEPTO DE EDUCACIÓN SOCIAL [ES]}

Si analizamos el concepto de educación desde la vertiente etimológica, observamos que da lugar a dos acepciones. La primera, alude a la educación como un proceso que se estimula desde fuera (educare). La segunda (ex-ducere), versa sobre la potencialidad interna del sujeto en la que se despliegan las disposiciones ya existentes y que la educación ha de estimular, motivar y alumbrar desde dentro, con tal fin. Los dos sentidos han luchado por imponerse a lo largo del tiempo, incluso en la ES.

\subsubsection{PROBLEMAS CONCEPTUALES}

El concepto de ES es polisémico. La diversidad de acepciones del término "social" complica el sentido de la adjetivación social que se asigna a la educación. Bajo la denominación de ES puede considerarse el amplio espectro de conexiones que, desde cualquier punto de vista, cabe establecer entre la sociedad, «lo social», y la educación; bien se considere a la sociedad como agente de la educación, o bien a la inversa, se aluda a la educación como condicionante de la sociedad (Medina Rubio, 1999).

La Educación Social presenta diversos aspectos y perspectivas. Destacamos, en esta ocasión, las dos que consideramos más significativas. Una, desde la vertiente normalizada: el proceso de socialización de los individuos, ese continuum que va desde la infancia a la edad adulta. Y otra, desde la óptica de la educación especializada, que hace referencia al tratamiento e intervención de todo tipo de inadaptados sociales.

Algunos autores, subrayan una $\mathrm{u}$ otra tendencia, señalando que lo importante en la tarea de la Educación Social consiste en,

Despertar el sentido de las relaciones (Kriekemans, 1968:129)

Necesidad de aprender roles sociales (Sansvisens, 1984:21)

Formar sentimientos, actitudes y conciencia de solidaridad (Quintana, 1984:169)

Concienciar a los individuos de su propia identidad y dignidad personal (Petrus, 1994:192)

La "Educación Social normalizada" es una inter- 
vención educativa que se hace con el fin de ayudar al individuo a que se realice en él, del mejor modo posible y sin desviaciones, el proceso de socialización. Es decir, la adaptación a la vida social y a sus normas, hasta alcanzar una alta capacidad de convivencia y de participación en la vida comunitaria (QUINTANA, 1984, p. 169). Este tipo de educación completa la educación general del individuo.

Según esta concepción, la ES se entiende como un aspecto importante de la educación general del ser humano. La correcta socialización exige la formación cívico-democrática con el fin de construir una ciudadanía responsable. Se trata de una educación para el desarrollo de valores sociales, orientada al fomento del comportamiento democrático de todos los ciudadanos, así como al compromiso de construcción social, cuyo objeto no es otro, que el logro de la madurez social del individuo.

La denomina "Educación Social especializada" hace referencia a un tipo de trabajo social de aspecto educativo que desempeña unas funciones pedagógicas. Se practica profesionalmente fuera de la escuela, pues no tiene que ver con el sistema educativo, sino, más bien, con la promoción del bienestar social. Pone en juego una serie de servicios sociales de carácter pedagógico que tienden, unas veces, a resolver problemas carenciales que sufren ciertos grupos sociales marginados, $y$, otras, a prevenir dichos problemas en la población general, asegurándole los medios (educación para la paz, ecológica, cívica, asociacionismo, voluntariado, servicios de tiempo libre, etc.) para llevar una vida comunitaria pletórica y correcta.

El primer tipo de ES es competencia de los educadores sociales ordinarios (familia y escuela) y se halla incluido dentro de los programas generales de educación. El segundo tipo, en cambio, es patrimonio profesional de los llamados «educadores sociales» y «pedagogos sociales». Los dos enfoques conviven en la actualidad.
Los profesionales que trabajan en el campo de lo social tienen por finalidad impulsar la participación del ser humano en la construcción de su propio futuro y el de la comunidad. Como expresan Sarrate, García y Pérez (2013, p. 1), no se puede olvidar que la Educación Social "está contribuyendo al desarrollo socioeducativo, así como a reforzar la identidad social y comunitaria".

\section{SEMEJANZAS Y DIFERENCIAS ENTRE CONCEPTOS AFINES: ES, TS, SE Y PS}

A continuación se estudian conceptos vinculados entre si y con la Pedagogía Social, como son: Educación Social, Trabajo Social y Sociología de la Educación

Por lo que se refiere a los aspectos que tienen en común se señalan algunos de los que consideramos más importantes. En primer lugar se alude al contexto de la acción social, como categoría relacional, en el que los profesionales de una y otra disciplina, realizan su tarea.

La intervención social requiere un trabajo en equipo, de carácter multidisciplinar, por lo que éstos suelen estar constituidos por profesionales especializados, a fin de estudiar la realidad social desde perspectivas disciplinares diferentes.

Conviene resaltar que cada profesional focaliza la acción social desde su formación especializada, lo que contribuye a enriquecer el trabajo en equipo, en orden a buscar formas de acercamiento, abordaje, investigación y conceptualización, de manera tal, que impriman nuevos sentidos y significados a la reflexión y a la acción, al mismo tiempo, que dan cuenta de sus diferencias específicas. Todo esto supone un esfuerzo serio, sistemático y metódico.

Los profesionales que trabajan en equipo asumen la propuesta de un enfoque transdisciplinar de la ciencia y de la intervención social. Tienen la posi- 
bilidad de trabajar metódica y sistemáticamente en orden a lograr una mejor solución de los problemas tanto desde la vertiente teórica como aplicada.

Existe una necesidad común a todas estas áreas sociales. Es urgente construir un vocabulario básico y adecuado, que contribuya a crear significados compartidos. Esto facilitará la creación de grupos de estudio, de discusión e investigación que conformarán un círculo de trabajo reflexivo sobre la acción. De este modo, se logrará que cada profesional encarne la especificidad y la diferencia de su trabajo y al mismo tiempo, sabrá dar razones de su existencia laboral.
Conviene reseñar, no obstante, que los profesionales que trabajan en el campo de lo social no suelen estar acostumbrados a sistematizar la experiencia cotidiana, ni a plasmar por escrito la reflexión sobre su acción. De este modo, no se posibilita la discusión epistemológica, ni el avance sobre la reflexión de los errores y/o equívocos; tampoco el análisis del proceso seguido para facilitar la identificación de los elementos de superación.

Se presentan a continuación, a modo de síntesis y de forma esquemática los elementos de concepto, objeto, método y ámbito- que configuran cada uno de los constructos señalados:

\begin{tabular}{|l|l|}
\hline \multicolumn{2}{|c|}{ EDUCACIÓN SOCIAL (ES) } \\
\hline Concepto & $\begin{array}{l}\text { La acción intencional y dinamizadora que provoca la progresiva configuración de la per- } \\
\text { sona en su contexto social; promueve su autonomía, integración y participación crítica, } \\
\text { constructiva y transformadora, a la vez que se ocupa de la reeducación, prevención y } \\
\text { terapia del conflicto y la inadaptación social. }\end{array}$ \\
\hline Objeto & Socialización \\
\hline Método & $\begin{array}{l}\text { Observación, entrevistas y estudio de casos, preferentemente. } \\
\text { Metodologías múltiples: tanto cualitativas como cuantitativas. }\end{array}$ \\
\hline Ámbitos & $\begin{array}{l}\text { Familia, Escuela y Educación extraescolar --> Educ. normalizada } \\
\text { Espacio no formal } \\
\text { Sectores marginales y problemáticos } \\
\text { Educación Especializada }\end{array}$ \\
\hline
\end{tabular}

\section{TRABAJO SOCIAL (TS)}

\begin{tabular}{|l|l|}
\hline Concepto & $\begin{array}{l}\text { La profesión del Trabajo Social promueve el cambio social, la solución de problemas en las } \\
\text { relaciones humanas y el fortalecimiento y la liberación de las personas para incrementar el } \\
\text { bienestar. Mediante la utilización de teorías sobre el comportamiento humano y los sistemas } \\
\text { sociales, el trabajo social interviene en los puntos en los que las personas interactúan con su } \\
\text { entorno. Los principios de los derechos humanos y la justicia social son fundamentales para } \\
\text { el trabajo social (FITS, 2000). }\end{array}$ \\
\hline Objeto & Ayuda social \\
\hline Método & Metodologías y técnicas múltiples de carácter preventivo, curativo y rehabilitador. \\
\hline
\end{tabular}




\begin{tabular}{|l|l}
\hline Ámbito & $\begin{array}{l}\text { Nivel micro: individuo y familia } \\
\text { N. medio: con grupos pequeños }\end{array}$ \\
N. macro: comunidades y organizaciones (también burocráticas).
\end{tabular}

\begin{tabular}{|l|l|}
\hline \multicolumn{2}{|c|}{ SOCIOLOGÍA DE LA EDUCACIÓN (SE) } \\
\hline Concepto & $\begin{array}{l}\text { Es una ciencia sociológica, teórico-aplicada. Entiende la educación en su dimensión social, } \\
\text { desde una perspectiva explicativa y descriptiva. }\end{array}$ \\
\hline Objeto & La relación entre Educación y Sociedad \\
\hline Método & Método sociológico. \\
\hline Ámbito & La Sociedad desde una perspectiva teórica, descriptiva y explicativa. \\
\hline
\end{tabular}

\begin{tabular}{|l|l|}
\hline \multicolumn{2}{|c|}{ PEDAGOGíA SOCIAL (PS) } \\
\hline Concepto & $\begin{array}{l}\text { Es una ciencia teórico-aplicada orientada a la mejora de los individuos, con un carácter pro- } \\
\text { positivo. Se ocupa de la prevención, la ayuda y reinserción de todos los que padecen algún } \\
\text { tipo de deficiencia o disocialización, orientada a la mejora de la calidad de vida desde una } \\
\text { perspectiva aplicada, tanto en la vertiente normalizada como en la especializada". }\end{array}$ \\
\hline Objeto & Educación Social. \\
\hline Método & $\begin{array}{l}\text { Metodología heurística cualitativa y cuantitativa. } \\
\text { Intervención social. }\end{array}$ \\
\hline Ámbito & $\begin{array}{l}\text { Familia y Escuela --> Educación normalizada } \\
\text { Espacio no formal } \\
\text { Sectores marginales y problemáticos } \\
\text { Educación Especializada }\end{array}$ \\
\hline
\end{tabular}

Este esfuerzo de reflexión que se presenta tiene por objetivo contribuir al diálogo y abrir nuevamente un debate sobre estos conceptos, tan necesitados de estudio y profundización y proyectar luz sobre los mismos. Ninguna ciencia, ni disciplina, llega a ser tal, si no es en relación con la totalidad de los círculos productores de conocimiento y saber. Estos conceptos se interrelacionan y complementan, por lo que las fronteras existentes entre ellos se desdibujan, lo que dificulta su delimitación conceptual.
La ciencia se va construyendo de modo progresivo, con carácter acumulativo, con la aportación de los que se dedican a la reflexión e investigación sobre la misma y el contraste con la práctica en los ámbitos cotidianos de acción social.

Desde esta perspectiva se hace una llamada a los distintos profesionales que actúan e intervienen en el espacio de lo social, para que consideren su trabajo, no 
sólo como un medio lícito de subsistencia, sino también como una posibilidad concreta de construcción metódica y sistemática de los objetos de estudio, investigación y actuación estratégicamente conducida.

\section{SÍNTESIS}

Las ciencias que se dedican al estudio de lo social parece que se revitalizan ante los grandes desafíos a los que se debe dar respuesta.

En este artículo se ha fundamentado la Pedagogía Social como ciencia y la Educación Social como ámbito de intervención. Los conceptos que se intentan delimitar son polisémicos y no tienen fronteras claras. La Pedagogía Social, tiene un cuerpo epistemológico que como cualquier otra ciencia se construye a la luz del marco histórico y socio-político que en cada etapa le toca vivir. Como ciencia se ha ido configurando en diálogo abierto con su contexto y en la discusión conceptual que mantiene con las ciencias afines.

La Pedagogía Social ha buscado, desde la Educación Social, responder a los retos que el individuo y la comunidad plantean a los problemas álgidos de cada época. Se va desarrollando en una doble dimensión: una Pedagogía Social, que aborda la dimensión integradora de lo epistemológico, lo tecnológico y lo normalizado y otra actuando en las situaciones de inadaptación, exclusión y conflicto social que daría lugar a una Pedagogía Social especializada.

El carácter científico de la Pedagogía social implica: ser normativa y prescriptiva, teórica y práctica. Tiene en cuenta las perspectivas de lo científico, lo disciplinar y lo profesional.

Por su parte, la Educación Social presenta dos vertientes significativas. Una, la normalizada que alude al proceso de socialización de los individuos. Y otra, la educación especializada, que hace referencia al tratamiento e intervención de todo tipo de inadaptados sociales.
Finalmente se recogen conceptos vinculados entre sí y afines a la Pedagogía Social para detectar sus semejanzas y diferencias.

La ciencia se va construyendo con carácter acumulativo y progresivo, con la aportación de los que se dedican a la reflexión e investigación sobre la misma y el contraste con la práctica en los ámbitos cotidianos de acción social.

\section{REFERENCIAS}

ANECA. Libro Blanco. Título de Grado en Pedagogía y Educación Social. V.1. Agencia Nacional de Evaluación de la Calidad y Acreditación. Madrid: Ministerio de Educación Cultura y Deporte, 2004.

ARROYO SIMON, M. ¿Qué es la Pedagogía social?. Bordón, 257, 1985, p.203-215.

BAS-PEÑA, E., PÉREZ-DE-GUZMÁN, V. Y VARGAS-VERGARA, M. Educación y Género: La formación de los educadores y educadoras sociales. Pedagogía Social. Revista Interuniversitaria, 2014, 23, p.95-120.

BÖMH, W. La Pedagogía Social en Alemania. En J. Ortega, Nuevos Retos de la Pedagogía Social. La formación del Profesorado. Salamanca: Sociedad Ibérica de Pedagogía Social, 2002.

CALIMAN, G. A Pedagogia Social na Itália. In: SILVA, J. Roberto da; SOUSA, J.C. de, y MOURA, R. (Eds). Pedagogia Social. São Paulo: Expressão e Arte, 2009.

CARIDE, J. L. Las fronteras de la Pedagogía Social. Barcelona: Gedisa, 2002.

FERMOSO, P. Pedagogía Social. Barcelona: Herder, 1994.

HÄMÄLÄINEN, J. The concept of social pedagogy in the field of social work. Journal of Social Work, 3, 2003, p.69-80. 
LEAHEY, T. H. Historia de la Psicología. Madrid: Prentice-Hall, 1998.

LUZURIAGA. L. Pedagogía social y Política. Buenos Aires: Losada, 1961.

MARÍN, R. PÉREZ, G. Pedagogía Social y Sociología de la Educación. Madrid: UNED, 1984.

MEDINA RUBIO, R. Educación Social y cambio de valores. Bortdón, 51, 4, 1999.

MOLLENHAUER, R. Einfuhrüng in die Sotzrial Pädagogik. Weinheim: Beitz Veriag, 1976.

MORADIELLOS. R. El oficio del historiador. Madrid: Siglo XXI, 1994.

NATORP, P. Pedagogía Social: Teoría de la Educación de la voluntad sobre la base de la Comunidad. Madrid: La lectura, 1913.

NOHL, H. Aufgaben und wege der sorial pádagogik. Weinheim: Beitz, 1965.

ORTEGA, J. Pedagogía Social especializada. Barcelona: Ariel, 1999.

PeREZ SerRANO, G. Pedagogía Social-Educación Social: Construcción Científica e intervención práctica. Madrid: Narcea, 2003.

PETRUS, A. El educador social y el perfil del educador social. In: SAEZ CARRERAS, J. (coord.). El Educador Social. Murcia: Universidad, 1994.
QUINTANA, J.M. Pedagogía Social. Madrid: Dykinson, 1984.

RÖHRS, H. Allgemeilne Erriehungswissenschaffe. Weinheim: Beitz, 1973.

ROSENDAL JENSEN, N. Will Social Pedagogiy become an Academic Discipline in Demark? In: KORBECK, j. JENSEN N. R. (eds). The Diversity of Social Pedagogy in Europe: Studies in Comparative Social Pedagogies and International Social Work and Social Policy. Bremen: Europäischer Hochschulverlag GmbH y Co. KG, 2009, p.189-210.

SÁEZ, J. La construcción de la Pedagogía Social: algunas vías de aproximación. In: PETRUS, A. (Coord.): Pedagogía Social. Barcelona: Ariel, 1997, p.40-66.

SANSVISENS, A. Introducción en la Pedagogía. Barcelona: Barcanova, 1984.

SARRATE CAPDEVILA, M. L.; GARCÍA LLAMAS, J. L. Y PÉREZ SERRANO, G. Exigencias profesionales del animador/a: Competencias clave. Pedagogía Social. Revista Interuniversitaria, 22, 2013, p.75-89.

TOURAINE, A. Un nuevo paradigma para comprender el mundo de hoy. Barcelona: Paidos, 2005.

TOURAINE, A. La mirada social: Un marco de pensamiento distinto para el siglo XXI. Barcelona: Paidos, 2009.

VOLPI, C. Tareas y funciones de la Pedagogía Social. Rev. Pedagogía Social, 1, 1986, p.29-38. 
Recebido: 22 de junho de 2014 Avaliado: 26 de junho de 2014 Aceito: 13 de agosto de 2014
1. Doctora em Ciencias de la Educación. Catedrática de Pedagogía Social. Universidad Nacional de Educación a Distancia (UNED). E-mail: gloriaperez@ edu.uned.es

2. Doctor en Ciencias de la Educación. Profesor Titular Metodología de la Investigación. Universidad Nacional de Educación a Distancia (UNED). E-mail: jlgarcia@edu.uned.es

3. Licenciada en Pedagogía. Becaria Formación Personal Investigador (FPI).

Universidad Nacional de Educación a Distancia (UNED). E-mail: anafernandez@ bec.uned.es 